\title{
Management of brain metastasis from eccrine porocarcinoma: illustrative case
}

\author{
Ryuichi Noda, MD, ${ }^{1}$ Tomohiro Inoue, MD, PhD, ${ }^{1}$ Sho Tsunoda, MD, ${ }^{1}$ Masafumi Segawa, MD, ${ }^{1}$ Yoshio Masuda, MD, ${ }^{3}$ \\ Teppei Morikawa, MD, $\mathrm{PhD},{ }^{3}$ and Atsuya Akabane, $\mathrm{MD}, \mathrm{PhD}^{2}$ \\ Departments of ${ }^{1}$ Neurosurgery, ${ }^{3}$ Diagnostic Pathology; and ${ }^{2}$ Gamma Knife Center, NTT Medical Center Tokyo, Shinagawa-ku, Tokyo, Japan
}

BACKGROUND Eccrine porocarcinoma (EP) is a rare malignant skin neoplasm, and there are still many unknowns regarding its natural history and treatment. Due to its scarcity, associated brain metastasis is a far rarer condition.

OBSERVATIONS A 71-year-old-woman with a history of EP was diagnosed with brain metastasis. In her clinical course, the patient underwent tumor removal surgery twice and Gamma Knife radiosurgery (GKRS) four times. The tumor showed a good response to radiotherapy. The histopathological findings of the brain tumor were consistent with those of the primary skin tumor.

LESSONS There are only a few case reports referring to the detailed treatment, especially with GKRS, of brain metastasis from EP. Few reports have presented a detailed histopathological comparison between the primary skin lesion and the metastatic brain lesion. Herein, the authors have described the clinical course, histological features, and results of multidisciplinary treatment for brain metastasis of EP.

https://thejns.org/doi/abs/10.3171/CASE21242

KEYWORDS eccrine porocarcinoma; brain metastasis; Gamma Knife radiosurgery

Eccrine porocarcinoma (EP) is a rare malignant skin neoplasm, accounting for approximately $0.005 \%-0.01 \%$ of all malignant skin tumors. $^{1}$ It is a malignant counterpart of poroma and was first described in 1967, ${ }^{2}$ and only 453 cases were reported until $2017 .^{3}$ It is often seen in the lower extremities of elderly patients aged $>60$ years. $^{3,4}$ Because of its scarcity, the natural course and treatment of EP are still unknown. Despite its highly invasive and potentially metastatic nature, there are very few reports of brain metastasis from EP. However, there is still no standard treatment for such brain metastasis. Thus, the treatment of EP, including the management of brain metastasis from EP, must be discussed in each case. Due to the lack of reports on the management of intracranial lesions related to EP, further accumulation of case reports and case series is required. We report a case of brain metastasis from EP, including its pathological findings and management, along with a literature review. The aim of this report is to help elucidate the clinical course, histopathological features, and treatment of brain metastasis from EP.

\section{Illustrative Case}

A 71-year-old-woman was referred to our institution's Department for Surgery. Her chief complaints were motor aphasia and dyslexia that gradually deteriorated 1 month before the presentation. The patient had a surgical history of EP of the scalp in the postauricular region and lung adenocarcinoma in situ, both resected 1 year before the presentation. The lung cancer was surgically cured, but the EP had a remnant lesion (22 $\mathrm{mm}$ in diameter) between the mastoid process and the styloid process, for which intensity-modulated radiation therapy (IMRT) was planned.

Further examination revealed a mass over the frontal lobe with a ring-enhanced pattern on contrast-enhanced magnetic resonance imaging (MRI) (Fig. 1A). On the basis of medical history and imaging results, brain metastasis was suspected. However, we could not determine whether the brain lesion was derived from the lung lesion or the remnant skin lesion.

The tumor was resected to alleviate the symptoms and confirm the histopathological diagnosis. Considering that the characteristics of brain

ABBREVIATIONS CK = cytokeratin; EP = eccrine porocarcinoma; GKRS = Gamma Knife radiosurgery; IMRT = intensity-modulated radiation therapy; MRI = magnetic resonance imaging; WBRT = whole-brain radiotherapy.

INCLUDE WHEN CITING Published June 28, 2021; DOI: 10.3171/CASE21242.

SUBMITTED April 19, 2021. ACCEPTED April 21, 2021.

(C) 2021 The authors, CC BY-NC-ND 4.0 (http://creativecommons.org/licenses/by-nc-nd/4.0/). 

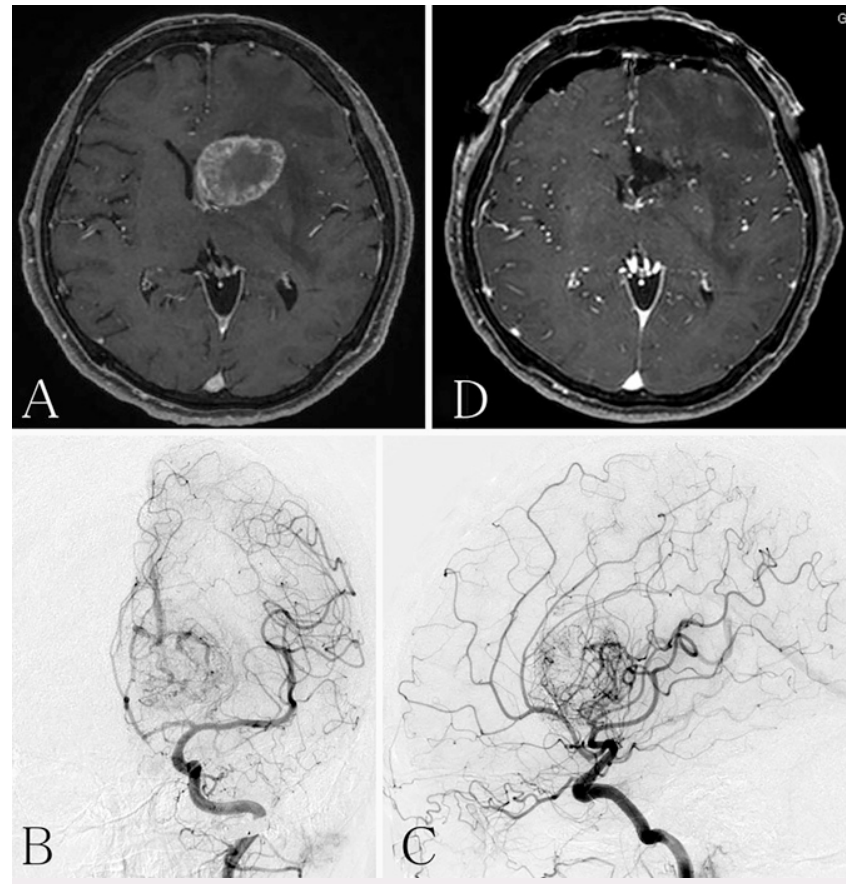

FIG. 1. Preoperative gadolinium-enhanced T1-weighted MRI image shows that the tumor was located on the caudate head and had a ringenhanced contrast pattern (A). The frontal view of the preoperative angiogram revealed hypervascularity of the tumor $(\mathbf{B})$. The lateral view of the preoperative angiogram revealed hypervascularity of the tumor (C). Postoperative MRI showed the gross total removal of the tumor (D).

metastasis from EP are still unknown, we performed angiography to determine the degree of vascularity. Angiographic results showed hypervascular staining of the tumor (Fig. 1B and C). Surgery was performed with the patient under general anesthesia. As expected from the highly vascularized feature on the preoperative angiography, we experienced some difficulty in achieving hemostasis during resection. We achieved gross total removal of the tumor using the interhemispheric approach (Fig. 1D). The histopathological diagnosis was compatible with brain metastasis from EP (Fig. 2). Regular contrast-enhanced MRI was performed for the postoperative evaluation. The patient also underwent IMRT for the remaining primary tumor in the postauricular area at another hospital immediately after the operation (70 Gy in 33 fractions). However, she did not receive any systemic chemotherapy.

Three months after surgery, follow-up MRI detected recurrent lesions near the tumor resection cavity (Fig. 3A). The first Gamma Knife radiosurgery (GKRS) was performed on recurrent lesions to regulate the tumor progression. Three lesions (on the left side of the lateral ventricle, the corpus callosum, and the left anterior horn of the ventricle) were detected and planned for treatment (target volume, $0.01-0.49 \mathrm{~mL}$; maximum dose, 26.7-33.8 Gy; marginal dose, 22.0 Gy). (Fig. 4) Brain MRI performed 1 month after the first GKRS demonstrated a dramatic reduction of the target lesion (Fig. 3B-D); however, new recurrent lesions were detected 5 months after the first GKRS. We conducted a second GKRS, and three lesions (two on the anterior falx and the other on the left anterior horn of the lateral ventricle) were included in the treatment plan (target volume, 0.01-1.2 mL; maximum dose, 38.5-42.8 Gy; marginal dose, 30-32.5 Gy, in five fractions). The three lesions were
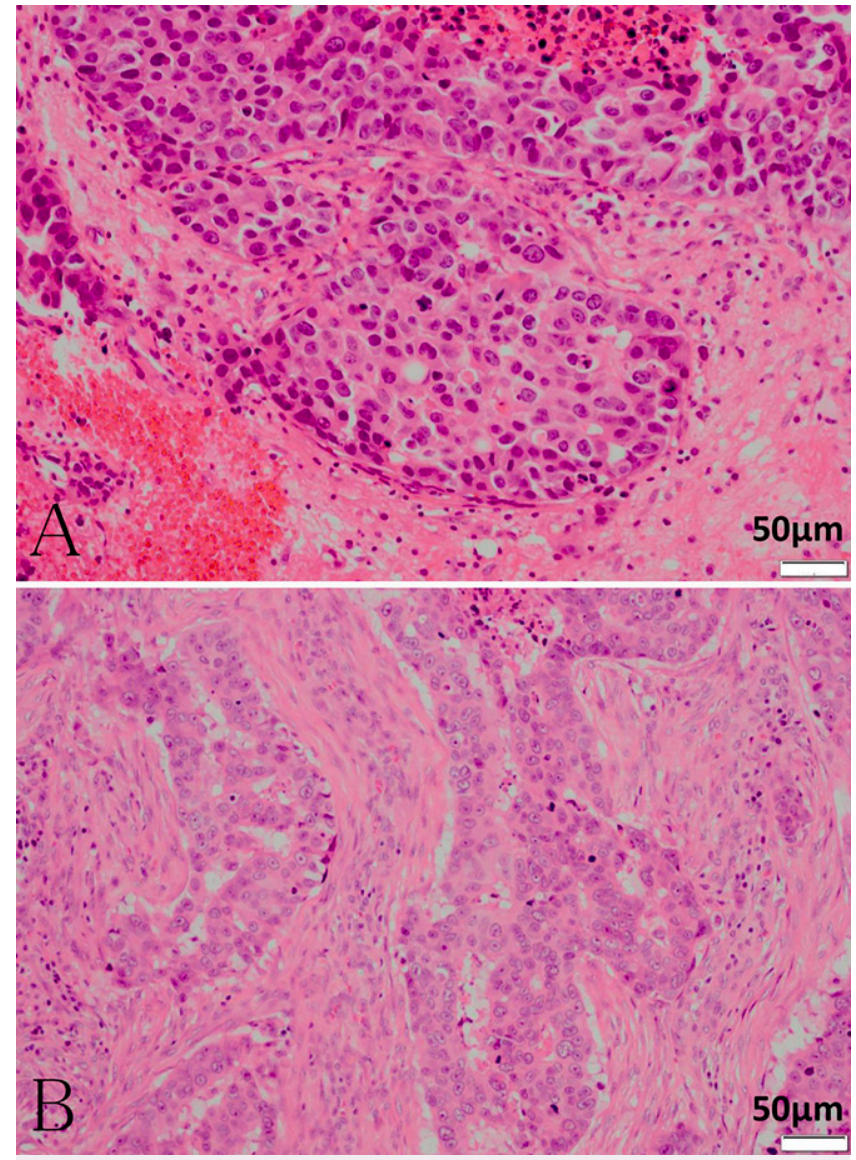

FIG. 2. Histopathology (hematoxylin and eosin stain) of the brain lesion (A) and the primary skin lesion (B). Ductlike configurations and atypical epithelial cells with prominent mitosis were seen in the EP and brain lesions (original magnification $\times 200$ ).

kept under control for 9 months until recurrence from two of the treated lesions, and these lesions were retreated by a third GKRS (target volume, 0.2-1.0 mL; maximum dose, 47.4 - 48.7 Gy; marginal dose, 35.1 Gy, in 13 fractions). Six months after the third GKRS, the treated lesion on the anterior falx showed rapid regrowth. Considering the tumor volume and the speed of growth, we decided to schedule a second tumor resection. Tumor resection was performed, and the pathological diagnosis of the resected recurrent tumor was compatible with regrowth of the EP brain metastasis. Two months after reoperation, the patient had a lumbar spinal cord metastasis at the L4-5 level and was treated with radiotherapy ( 30 Gy in 10 fractions). After 5 months, MRI revealed four novel brain metastases located in the fourth ventricle, the left thalamus, the left side of the anterior horn of the lateral ventricle, and the choroid plexus, respectively. The fourth GKRS (target volume, $0.04-0.7 \mathrm{~mL}$; maximum dose, 46.7-50.0 Gy; marginal dose, 35 Gy in 10 fractions) was performed. MRI performed after the fifth fraction revealed a new lesion in the internal auditory canal. The lesion was included in the treatment plan and was treated with the next five fractions (target volume, $0.02 \mathrm{~mL}$; maximum dose, $40.0 \mathrm{~Gy}$; marginal dose, $30 \mathrm{~Gy}$ ). Most GKRS sessions showed a good treatment response.

Although the lesions treated by the fourth GKRS showed a good response, a 3-month follow-up MRI scan showed new metastasis in the cavernous sinus and signs of progressive dissemination to the 

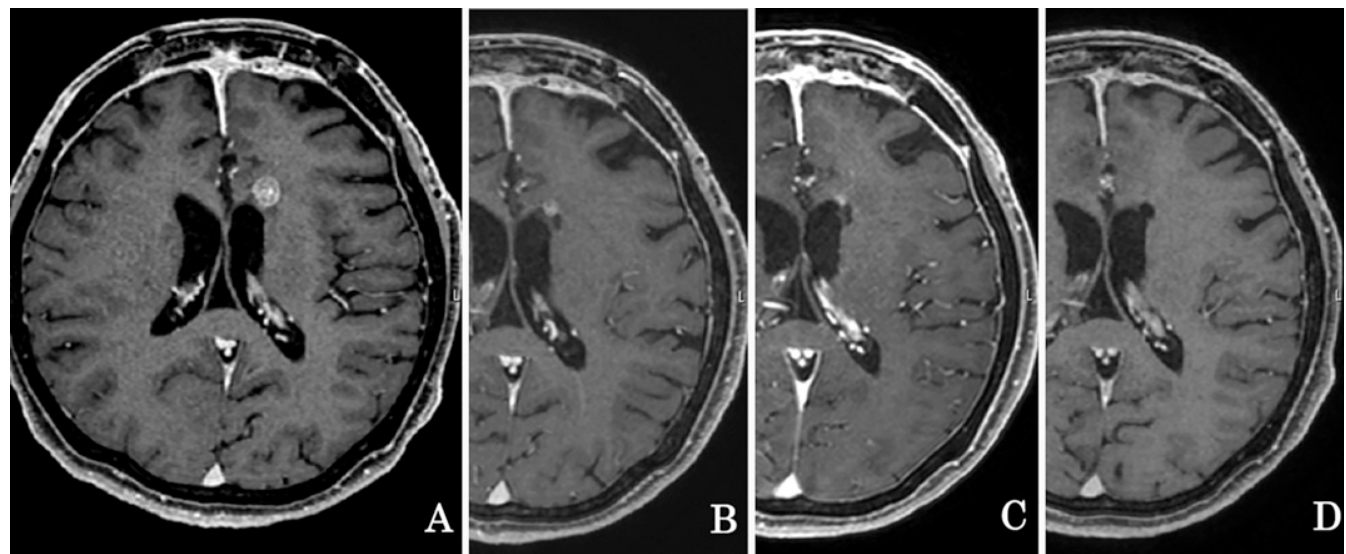

FIG. 3. Follow-up MRI after the first GKRS session showed tumor regression, and obliteration of the tumor was achieved on MRI in the 5th month. MRI before GKRS (A), 1 month after GKRS (B), 4 months after GKRS (C), and 5 months after GKRS (D).

central nervous system. Whole-brain radiotherapy (WBRT) was proposed as an option, but the patient refused further treatment and was provided palliative care.

\section{Discussion}

\section{Observations}

EP is a rare malignant skin neoplasm. A European study reported an incidence rate of $<0.28 / 100,000,{ }^{5}$ and another study from the United States reported an age-adjusted incidence rate ratio of 0.4 cases per 1 million person-years. ${ }^{6}$ Currently, there are no established treatment strategies for metastatic EP, and a chemotherapeutic protocol does not exist. Although some case reports and series have reported the use of preexisting chemotherapeutics, most authors have admitted the relative chemoresistance of $E P^{7}$ thus questioning its effectiveness. Due to the poor prognostic characteristics, there are very few reports mentioning the management of brain metastases from EP. To the best of our knowledge, only three case reports have referred to brain metastases from $\mathrm{EP}^{8-10}$ but none of them have highlighted the detailed management (Table 1). In our study, the patient underwent resection, which achieved gross total resection, followed by four sessions of GKRS, which were effective, and an additional open resection for recurrent lesions.

On the basis of the pathological aspect, because the patient had a past history of lung cancer, we had to confirm that the brain lesion was derived from EP by histopathological examination of both the lung and skin specimens. The morphological features of the brain tumor (Fig. 2A) were apparently different from those of the lung cancer and corresponded to those of the skin lesion (Fig. 2B). In addition, we performed immunostaining of the primary skin lesion to confirm the diagnosis of the skin lesion as EP. Cytokeratin 7 (CK7) staining, known to be reactive to $E P,{ }^{11}$ was positive, and CK5 and p40 staining were nonreactive. The immunohistochemical results were compatible with EP.
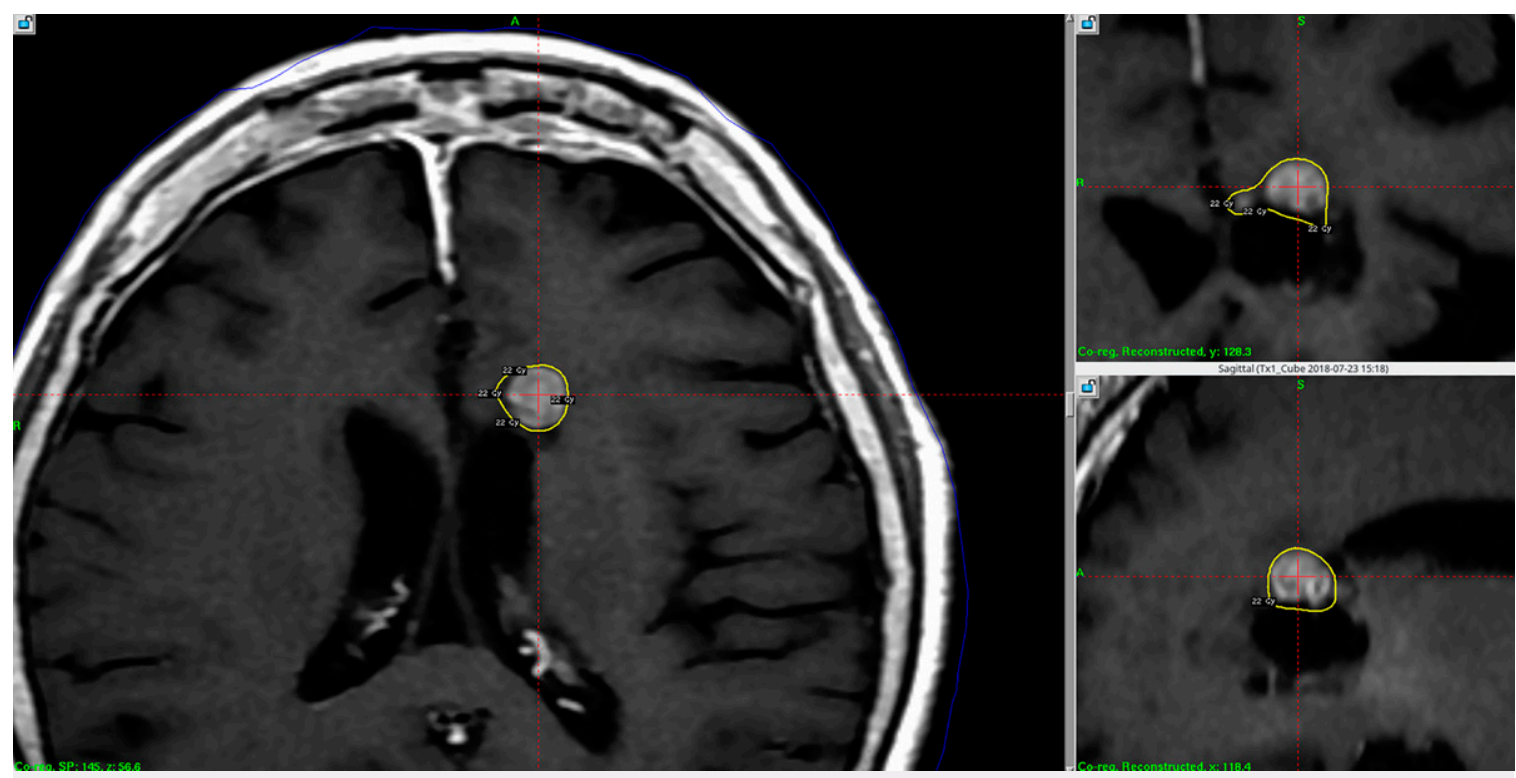

FIG. 4. The treatment plan of the first GKRS targeted at the lesion on the left side of the lateral ventricle (anterior horn). The tumor volume was $0.49 \mathrm{~mL}$, the maximum dose was $33.8 \mathrm{~Gy}$, and the marginal dose was $22.0 \mathrm{~Gy}$ (yellow outlined areas). 
TABLE 1. Previous reports of brain metastasis from EP

\begin{tabular}{lccccccc}
\hline \multicolumn{1}{c}{ Authors \& Yrs } & Sex & Age $($ Yrs $)$ & Primary Site & Tx for Primary Site & $\begin{array}{c}\text { Tx for Brain } \\
\text { Metastasis }\end{array}$ & Follow-Up & Outcome \\
\hline Kim et al., $2007^{8}$ & M & 42 & Rt palm & Surgery; RT & GKRS & 7 yrs & Palliative care \\
\hline Thibodeau et al., $2018^{9}$ & M & 64 & Lt scalp & Surgery; RT & Surgery & 29 mos & Died of disease \\
\hline Lee et al., $2019^{10}$ & F & 67 & Lt lower leg & $\begin{array}{c}\text { Surgery; chemotherapy; } \\
\text { RT; immunotherapy }\end{array}$ & Cyberknife & 4 yrs & Complete remission \\
\hline Present case & F & 71 & Lt scalp & Surgery; IMRT & Surgery; GKRS & 4 yrs & Palliative care \\
\hline
\end{tabular}

$\mathrm{RT}=$ radiotherapy; $\mathrm{Tx}=$ treatment.

The pathological characteristics proved that the brain lesion was derived from EP but not from lung cancer. The resected recurrent tumor also showed correspondence to EP in terms of the morphological features and ruled out radiation-related necrosis. There is only one report comparing the pathological findings between a primary EP and the associated brain lesions; ${ }^{9}$ our case is the second one to report such characteristics, including the histopathological picture of a recurrent brain lesion. Histopathological examination of the resected tumor helped in identifying the primary lesion on the basis of pathological characteristics.

This is the second case report of the use of GKRS for brain metastasis from EP. Although there are two case reports of brain metastasis from EP treated by stereotactic radiosurgery, ${ }^{8,10}$ none of them has referred to the details, including the treatment response. In our case, most of the lesions showed a significant response to GKRS, except for the recurrent lesion, which was resected in the second surgery. In our case, most of the recurrent lesions emerged from the area adjacent to the tumor cavity. This could have been caused by a potential occult residual tumor hidden inside the margin of the tumor cavity.

On the one hand, in a randomized trial by Patchell et al., compared with surgery alone, a combination of surgery and postoperative WBRT was found to reduce recurrence and death of neurological causes. ${ }^{12}$ On the other hand, a randomized trial by Kayama et al. revealed that there was no significant difference in overall survival following adjunctive WBRT and salvage stereotactic radiosurgery after tumor resection. ${ }^{13}$ Thus, to avoid late cognitive dysfunction, we did not choose postoperative WBRT, but we performed salvage stereotactic radiosurgery for the recurrent lesions. However, on the basis of our experience of multiple recurrences from the tumor cavity, prophylactic GKRS to the tumor bed could have been an alternative management strategy. ${ }^{14}$

This is the first report to describe the details and effectiveness of GKRS for brain metastases of EP. Besides, the effectiveness of GKRS has been proved for metastatic brain tumors of common organs. ${ }^{15}$ Our case showed the possibility of another indication. In addition, the favorable results of IMRT performed on the remaining primary lesion in our case also implied the possible high radiosensitivity of this cancer.

\section{Lessons}

There are no guidelines for the treatment or follow-up of EP. This report aimed to show the clinical course, histological features, and results of multidisciplinary treatment for brain metastases of EP. Moreover, this report may contribute to the development of a standard multimodal treatment strategy for metastatic EP in the future.

\section{Acknowledgments}

We thank Dr. Hiroki Mitani from the Department of Head and Neck, Cancer Institute Hospital of the Japanese Foundation for Cancer Research, for generously providing us with the patient information.

\section{References}

1. Wick MR, Goellner JR, Wolfe JT III, Su WP. Adnexal carcinomas of the skin. I. Eccrine carcinomas. Cancer. 1985;56(5):1147-1162.

2. Pinkus $H$, Mehregan $A H$. Epidermotropic eccrine carcinoma. A case combining features of eccrine poroma and Paget's dermatosis. Arch Dermatol. 1963;88:597-606.

3. Salih AM, Kakamad FH, Baba HO, et al. Porocarcinoma; presentation and management, a meta-analysis of 453 cases. Ann Med Surg (Lond). 2017;20:74-79.

4. Robson A, Greene J, Ansari N, et al. Eccrine porocarcinoma (malignant eccrine poroma): a clinicopathologic study of 69 cases. Am J Surg Pathol. 2001;25(6):710-720.

5. Marone U, Caracò C, Anniciello AM, et al. Metastatic eccrine porocarcinoma: report of a case and review of the literature. World $J$ Surg Oncol. 2011;9:32.

6. Blake PW, Bradford PT, Devesa SS, Toro JR. Cutaneous appendageal carcinoma incidence and survival patterns in the United States: a population-based study. Arch Dermatol. 2010;146(6):625-632.

7. De luliis $F$, Amoroso L, Taglieri L, et al. Chemotherapy of rare skin adnexal tumors: a review of literature. Anticancer Res. 2014;34(10): 5263-5268.

8. Kim JW, Oh DJ, Kang MS, et al. A case of metastatic eccrine porocarcinoma. Acta Derm Venereol. 2007;87(6):550-552.

9. Thibodeau ML, Bonakdar M, Zhao E, et al. Whole genome and whole transcriptome genomic profiling of a metastatic eccrine porocarcinoma. NPJ Precis Oncol. 2018;2(1):8.

10. Lee KA, Cioni M, Robson A, Bataille V. Metastatic porocarcinoma achieving complete radiological and clinical response with pembrolizumab. BMJ Case Rep. 2019;12(9):e228917.

11. Anubha B. Transmutation of sweat glands - eccrine porocarcinoma. J Clin Diagn Pathol. 2019;1(1):19-27.

12. Patchell RA, Tibbs PA, Regine WF, et al. Postoperative radiotherapy in the treatment of single metastases to the brain: a randomized trial. JAMA. 1998;280(17):1485-1489.

13. Kayama T, Sato $S$, Sakurada K, et al. Effects of surgery with salvage stereotactic radiosurgery versus surgery with whole-brain radiation therapy in patients with one to four brain metastases (JCOG0504): a phase III, noninferiority, randomized controlled trial. J Clin Oncol. 2018;36(33):3282-3289.

14. Iorio-Morin C, Masson-Côté L, Ezahr Y, et al. Early Gamma Knife stereotactic radiosurgery to the tumor bed of resected brain metastasis for improved local control. J Neurosurg. 2014;121(suppl): 69-74.

15. Yamamoto M. Gamma Knife radiosurgery: technology, applications, and future directions. Neurosurg Clin N Am. 1999;10(2):181-202. 


\section{Disclosures}

The authors report no conflict of interest concerning the materials or methods used in this study or the findings specified in this paper.

\section{Author Contributions}

Conception and design: Noda, Inoue, Akabane. Acquisition of data: Noda, Masuda, Morikawa. Analysis and interpretation of data: Noda. Drafting the article: Noda, Inoue. Critically revising the article: Inoue, Morikawa, Akabane. Reviewed submitted version of manuscript: Inoue, Tsunoda, Morikawa, Akabane. Approved the final version of the manuscript on behalf of all authors: Noda. Administrative/technical/ material support: Segawa. Study supervision: Inoue, Akabane.

\section{Correspondence}

Ryuichi Noda: NTT Medical Center Tokyo, Tokyo, Japan. rnrn46_8447@yahoo.co.jp. 